\title{
Evaluation of cutaneous flap survival by IR thermography
}

Petar Drvis ${ }^{1}$, Drazen Shejbal ${ }^{1}$, Srecko Svaic², Ivanka Boras², Igor Sundov $^{2}$, Mirela Susa², Vladimir Bedekovic ${ }^{1}$, Livije Kalogjera ${ }^{1}$, Igor Petrovic $^{3}$, Predrag Sikiric ${ }^{3}$

${ }^{1}$ Department of Otolaryngology - Head and Neck Surgery, University Hospital »Sestre milosrdnice«, Zagreb, Croatia

${ }^{2}$ Faculty of Mechanical Engineering and Naval Architecture, University of Zagreb, Zagreb, Croatia

${ }^{3}$ Department of Pharmacology and Pathology, Faculty of Medicine, Universitiy of Zagreb, Zagreb, Croatia

Contact: Petar Drvis, MD, Department of Otolaryngology - Head and Neck Surgery, University hospital »Sestre milosrdnice«, Vinogradska c. 29, 10000 Zagreb, Croatia

Phone: **385912500265

E-mail: petar100@,vip.hr

\begin{abstract}
:
There are plenty of recent studies showing the influence of different drugs on the survival of the cutaneous flap. The effects of a topically applied capsaicin, methylprednisolon, mitomycin and gastric pentadecapeptide BPC-157 in improving skin vitality and preventing distal flap necrosis were tested in a random-pattern dorsal skin flap model.
\end{abstract}

Wistar rats were randomized into five groups, four experimental groups and a control group. A standardized full thickness dorsal random-pattern skin flap was raised on each rat and sutured back into place. A gelatin sponge was placed before suturing between the flap and its recipient bed, with $0.9 \%$ saline in the control group and with capsaicin, methylprednisolon, mitomycin and pentadecapeptide BPC157 in the experimental groups. The flap survival was judged one 
week postoperatively, and the extent of skin flap survival was compared between the experimental groups and control. Vitality of the flap and survival area was measured by infrared termography . Infrared termography is non-invasive diagnostic method which offers two-dimensional representation of the surface temperature of the skin. It is useful for the evaluation of cutaneous blood circulation of the skin and superficial temperature can be an indicator for the vitality of the skin flap.

The topically applied methylprednisolon and pentadecapeptide BPC-157 resulted in a statistically significant decrease in skin flap necrosis, compared with the control group ( $\mathrm{p}<0.05)$, topically applied capsaicin and mitomycin did not show statistically significant decrease in skin flap necrosis. The topically applied methylprednisolon and pentadecapeptide BPC-157 was effective in reducing ischemic necrosis in failing random-pattern skin flaps in this rat model.

The results of this study suggest that such a topical drug application might have significant effects in the reduction of ischemic necrosis in the distal parts of skin flaps, and this treatment might also have applications as prophylactic therapy for risky skin flaps. Thermography of the skin is an easy method for estimating the vitality and survival area of the skin flaps.

\section{Key-words:}

Cutaneous flap survival, infrared thermography 


\section{Introduction:}

Flap is a part of the tissue divided from it's background, used for covering the tissue defect in plastic and reconstructive surgery. Flap necrosis is a significant complication in all types of flap surgery. Many recent studies show the influence of different drugs on the survival of the cutaneous flap. The results of these studies suggest that topical application of certen drugs might have significant effects in the reduction of ischemic necrosis in the distal parts of skin flaps. Some studies show that topical application of capsaicin result in a significant increase of flap survival area. Pretreatment with capsaicin, which depletes neuropeptide transmitter content from primary sensory neurons, has controversal influence. In some studies it showed a significant decrease in flap survival area compared to normal animals, and in the others capsaicin-pretreatman showed increased vascularity and flap survival or it showed no difference in flap survival between experimental groups and controls . Corticosteroid treatment can improve the survival of surgical skin flaps. Venous flaps of the animals receiving daily methylprednisolone injections were noted to have statistically significantly improved venous flap survival, compared to the control group. Mitomycin is effective in depressing wound healing. Gastric pentadecapeptide BPC-157 improves burn wound healing or bone fracture healing. There wasn' t found any available literature for the influence of the mitomycin or pentadecapeptide BPC-157 on the cutaneus flap 
vitality, so our aim was to investigate the influence of these drugs on cutaneous flap survival.

Infrared thermography measures infrared radiation from an object. The radiation measured by the camera depends on the object temperature, ambient temperature and objects emissivity, which is one of the most important parametars in thermography. Human and shaved rat's skin exhibits an emissivity close to 1 . So far has been evaluated the applicability of various thermografic techniques in diagnosis of radiation and burn injury, early diagnosis of cancer or evaluation of cutanous flap survival. Temperature distribution of the skin can be closely related to the arterial blood supply. Superficial temperature as an indicator of cutaneous blood circulation can be visualized easily by thermography (Fig.1).

The effects of a topically applied capsaicin, methylprednisolon, mitomycin and gastric pentadecapeptide BPC-157 in improving skin vitality and preventing distal flap necrosis were tested in a randompattern dorsal skin flap model. 


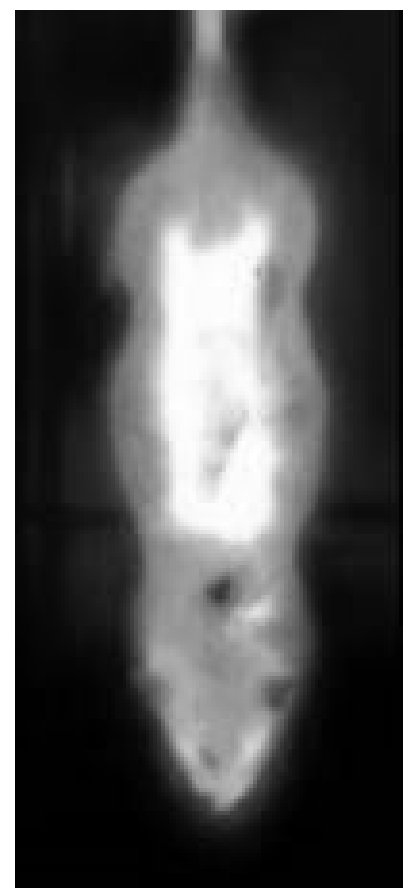

Fig. 1: Thermografic image of the rat cutaneous flap

\section{Materials and methods:}

Local Ethical Committee approved experimental protocol. Fifty female Wistar rats, weighting between 200 and $250 \mathrm{~g}$ were randomized into five groups of 10 animals, four experimental groups and a control group. The dorsaal skin of the rat was shaved. A standardized full thickness inferiorly based dorsal random-pattern skin flap (10.0 × 2.0 $\mathrm{cm}$ ) was raised on each rat and sutured back into place. A gelatin sponge was placed before suturing between the flap and its recipient bed, soaked with $0.9 \%$ saline in the control group and with capsaicin (0.2\%), methylprednisolon (30 mg/kg), mitomycin $(0.2 \mathrm{mg} / \mathrm{ml})$ and pentadecapeptide BPC-157 $(2 \mu \mathrm{g} / \mathrm{ml})$ in the experimental groups. The flap survival was judged one week postoperatively, and the extent of 
skin flap survival was compared between the experimental groups and control. Vitality of the flap and survival area was measured by infrared termography. Measurment was made by thermographic system ThermaCAM SC2000 (Fig. 2). The computorised image analysis was made by ThermaCAM Researcher 2002. Statistical analysis was made by Mann Whitney test for diferences beetween the analysed group and the controle group (saline).

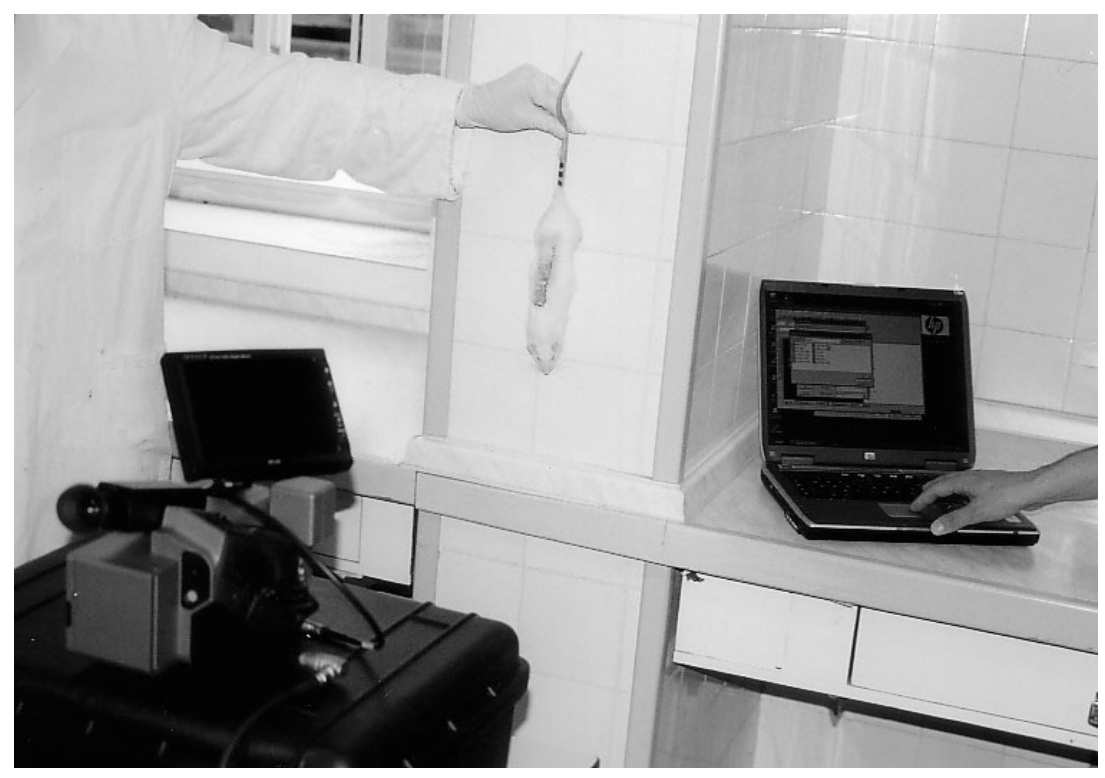

Fig. 2: Measurment by thermographic system ThermaCAM SC2000

\section{Results:}

The topically applied methylprednisolon and pentadecapeptide BPC157 was effective in reducing ischemic necrosis in failing random- 
pattern skin flaps, it resulted in a statistically significant decrease in skin flap necrosis, compared to the control group $(\mathrm{p}<0.05)$ and topically applied capsaicin and mitomycin did not show statistically significant decrease in skin flap necrosis, measured by infrared thermography one week postoperatively (Fig 3).

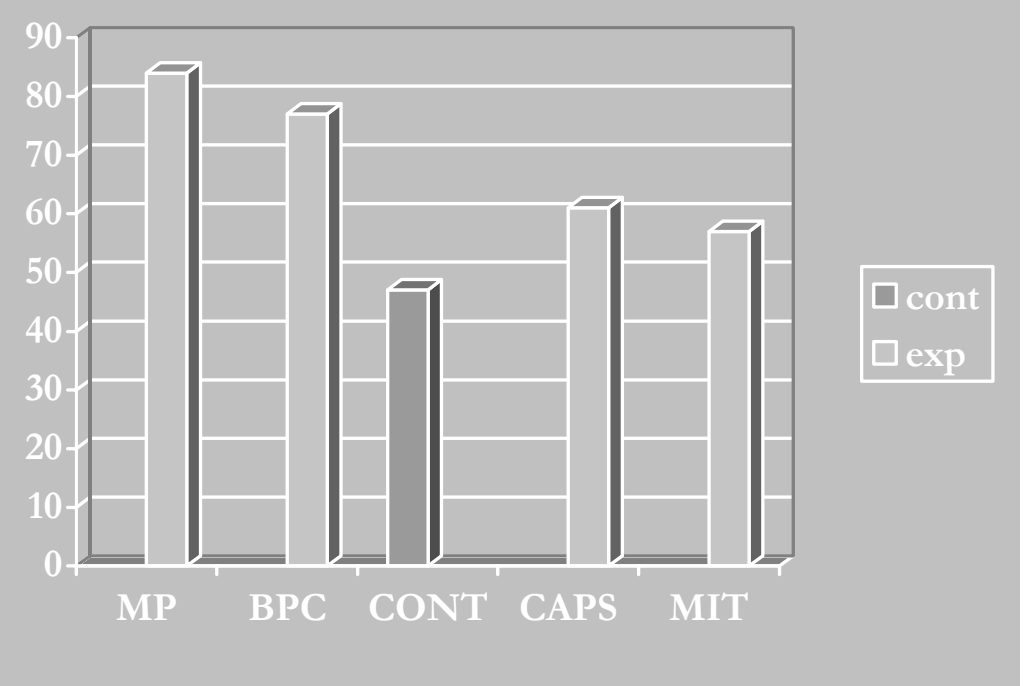

Fig. 3. Cutaneous flap survival in examined group (in milimeters): MP - methylprednisolon, BPC - pentadecapeptide BPC-157, CONT control (saline), CAPS - capsaicin, MIT - mitomycin.

\section{Conclusion:}

The topically applied methylprednisolon and pentadecapeptide BPC157 was effective in reducing ischemic necrosis in failing randompattern skin flaps in this rat model. The results of this study suggest that such a topical drug application might have significant effects in 
the reduction of ischemic necrosis in the distal parts of skin flaps, and this treatment might also have applications as prophylactic therapy for risky skin flaps in plastic and reconstructive surgery.

Infrared thermography is non-invasive diagnostic method which offers two-dimensional representation of the surface temperature of the skin and it is a sensitive method to detect altered tempetarure distribution expressed through the histograms, evaluating the vitality of the skin flap by computorised image analysis. Sufficient blood supply of the flap is a significant factor for proper wound healing and cutaneous flap survival. Thermography of the skin is an easy method for estimating the blood circulation of the skin flap, but it is limited to hair-free or shaved skin areas.

\section{References:}

1. IinumaT,SawadaY. Topical application of capsaicin and flap survival. Br J Plast Surg 1996;49:319-20

2. Miawaki T, Jackson IT, Bier UC, Andrus L, Williams F, Bradford M. The effect of capsaicin ointment on skin for the survival of a cutaneous flap. Eur J Plast Surg 2001;24:28-30

3.

Chang CC, Maruyama Y, Inomata K. Increased flap survival after sensory denervation with capsaicin accompanied by delay. J Reconstr Microsurg 1990 ;6(1):29-33

4. Westin M, Heden P. Sensory denervation with capsaicin does not influence the critical ischemia time in rat island flaps. Scand J Plast Reconstr Surg Hand Surg 1989; 23:173-6

5. Saray A, Can B, Sevin K. Effects of methylprednisolone on the viability of experimental flow-through venous flaps. J Reconstr Microsurg $2002 ; 18: 615-22$

6. Demir T, Turgut B, Akyol N, Ozercan I, Ulas F, Celiker U. Effects of amniotic membrane transplantation and mitomycin $\mathrm{C}$ on wound healing in experimental glaucoma surgery. Ophthalmologica 2002; 216: 438-42 
7.

Rahbar R, Jones DT, Nuss RC, Robertson DW, Kenna MA, McGill TJ, Healy GB. The role of Mitomycin in the prevention and treatment of scar formation in the pediatric aerodigestive tract. Arch Otolaryngol Head Neck Surg. 2002; 128: 401-406

8.

Sikiric P, Seiwerth S, Mise M, Staresinic M, Bedekovic V, Zarkovic N, Borovic S, Gjurasin M, Boban-Blagaic A, Blagaic L, Rucman N, Anic T. Corticosteroid-impairment of healing and gastric pentadecapeptide BPC-157 cream in burned mice. Burns 2003; 29: 323-334

9. Koteles GJ, Benko I, Nemeth G. Use of termography in diagnosis of local radiation injuries. Health Physics, 74, 1998; p 264265

10. Eisenbeis W, Marotz J, Schrade JP. Reflection-optical multispectral imaging method for objective determination of burn depth. Burns. 1999;25, p 697-704

11. Pau HW, Sievert U, Wild W. Thermography of the skin covering a cochlear implant - temperature as an indicator for blood circulation Laryngorhinootologie. 2003 ;82(9):615-9.

12. Parisky YR, Sardi A, Hamm R, Hughes K, Esserman L, Rust S, Callahan K. Efficacy of computerized infrared imaging analysis to evaluate mammographically suspicious lesions. AJR Am J Roentgenol. 2003;180:263-9

Bulstrode NW, Wilson GR, Inglis MS. No-touch free-flap temperature monitoring. Br J Plast Surg. 2002 Mar;55(2):174.

14. Chijiwa T, Arai K, Miyazaki N, Igota S, Yamamoto N. Making of a facial perforator map by thermography. Ann Plast Surg. $2000 ; 44: 596-600$.

15. Buemi M, Vaccaro M, Sturiale A, Galeano MR, Sansotta C, Cavallari V, Floccari F, D'Amico D, Torre V, Calapai G, Frisina N, Guarneri F, Vermiglio G. Recombinant human erythropoietin influences revascularization and healing in a rat model of random ischaemic flaps. Acta Derm Venereol. 2002;82(6):411-7. 
http://dx.doi.org/10.21611/qirt.2004.047 